\title{
Impact-Induced Delamination Detection of Composites Based on Laser Ultrasonic Zero-Lag Cross-Correlation Imaging
}

\author{
Yun-Kyu An \\ Department of Architectural Engineering, Sejong University, Seoul 05006, Republic of Korea \\ Correspondence should be addressed to Yun-Kyu An; yunkyuan@sejong.ac.kr
}

Received 17 July 2016; Revised 19 September 2016; Accepted 28 September 2016

Academic Editor: Ying Wang

Copyright (C) 2016 Yun-Kyu An. This is an open access article distributed under the Creative Commons Attribution License, which permits unrestricted use, distribution, and reproduction in any medium, provided the original work is properly cited.

\begin{abstract}
This paper presents impact-induced delamination visualization by zero-lag cross-correlation (ZLCC) imaging computed using fully noncontact laser scanned ultrasonic wavefields. The proposed technique enables instantaneous visualizing of invisible delamination of composite materials without any sensor installation. Moreover, it provides robust damage diagnosis without comparing with baseline data obtained from the undamaged condition of a target structure, making it possible to minimize false alarms. First, the existence of internal delamination-induced standing waves is proven by employing a finite element analysis. Then, how ZLCC can physically isolate and visualize only the standing wave feature from the measured ultrasonic wavefields is shown. To experimentally validate the proposed technique, a fully noncontact laser ultrasonic imaging system is introduced, and the internal delamination is visualized by laser scanning in a graphite fiber composite plate. The experimental results reveal that hidden delamination is successfully and automatically visualized and quantified without any users' intervention.
\end{abstract}

\section{Introduction}

Composite materials have gained popularity for various structures of aerospace and civil and mechanical fields, because they have many advantages such as lightweight and higher strength over other existing materials. For example, composite materials corresponding to more than $20 \%$ of total weight are used for Air Bus 380 and more than $40 \%$ are used for Boeing 787. However, these composite materials typically have higher brittleness than metals and are intrinsically susceptible to external bending and shear forces between laminated layers. Aircraft structures are often subjected to these external forces such as a bird strike, cyclic loading under inservice conditions, and other abrupt impact loads, resulting in delamination between internal layers. The most challenging issue for delamination detection is that typical delamination cannot be observed by naked eyes on the structure surface even though it seriously deteriorates the strength of composite structures. Thus, a number of nondestructive testing (NDT) techniques have been adopted to tackle the issue. One of the widely accepted NDT techniques is an ultrasonic NDT technique. Zhao et al. conducted experiments on a full-scale aircraft composite wing for damage identification and localization using a lead zirconate titanate (PZT) sensor array [1]. Di Scalea et al. studied the monitoring of the composite wing skin-to-spar joint in an unmanned aerial vehicle using guided waves obtained from a pair of macrofiber composites [2]. More recently, An et al. investigated the feasibility of the integrated impedance and guided wave technique for monitoring of a full-scale aircraft wing structure under temperature and external loading variations [3].

However, these conventional approaches often suffer technical challenges from the use of contact-type transducers. First, spatially limited ultrasonic responses obtained from sensors installed at several discrete points may not achieve spatial resolution high enough to detect small incipient damage. Second, damage localization may not be accomplished using spatially limited sensors. Third, installed transducers, electric wires, and the associated onboard data acquisition devices not only augment the weight of a target structure but also increase the system complexity. Furthermore, the repair or replacement with respect to malfunctioned transducers permanently installed on a target structure is a challenging task. 
To overcome these technical limitations, noncontact NDT techniques are strongly desired to be adopted. In particular, fully noncontact laser ultrasonic imaging (LUI) techniques have been extensively studied as emerging damage detection techniques with the remarkable development of laser technology and the corresponding measurement devices. The advantages of the LUI technique are that (1) ultrasonic wavefield images with high spatial and temporal resolutions are constructed without any sensor installation, providing intuitive damage diagnosis; (2) damage diagnosis can be performed without relying on baseline data obtained from the pristine condition of a target structure, enabling it to be less vulnerable to false alarms due to environmental and operational variations; and (3) it is nonintrusive, costeffective, rapidly deployable, and applicable to harsh environments such as high temperature and radioactive conditions.

With these advantages, a number of fully noncontact LUI techniques have been recently developed. Dhital and Lee developed a fully noncontact LUI technique using a Qswitched pulsed laser for ultrasonic generation and an air coupled transducer (ACT) for ultrasonic measurement [4]. An et al. proposed a complete noncontact LUI technique by combining a Q-switched pulsed laser for ultrasonic generation and a laser Doppler vibrometer (LDV) for ultrasonic measurement and demonstrated hidden crack visualization on an aluminum plate [5]. Such complete noncontact LUI system has been also used for internal damage detection in composite structures. Chia et al. proposed an adjacent wave subtraction method for internal defect detection in a composite wing structure [6], and Park et al. visualized internal delamination in composite structures [7]. Then, Harb and Yuan also developed a fully noncontact system by integrating ACT for ultrasonic generation with LDV for Lamb wave characterization [8]. However, the development of rapid and computational cost-effective imaging techniques is still necessary.

To come up with the demand, the zero-lag cross-correlation (ZLCC) imaging technique using fully noncontact LUI technique is proposed for internal delamination visualization in a composite plate in this study. To achieve it, the ultrasonic wave interactions with an internal delamination are thoroughly investigated by employing a finite element (FE) method. Based on delamination-induced standing waves identified as a unique feature representing the delamination existence and location, a ZLCC imaging technique is theoretically developed to isolate and visualize only the standing wave components from the measured ultrasonic wavefields. Finally, the proposed technique is experimentally validated by visualizing hidden delamination of a composite plate using the LUI system.

This paper is organized as follows. First, the existence of standing waves generated by an internal delamination is investigated through the FE analysis in Section 2. Then, the ZLCC imaging technique is theoretically developed in Section 3. In Section 4, a target composite specimen with an internal delamination and the LUI system are described for the experimental validation. The corresponding experimental results are shown in Section 5. Finally, this paper concludes with brief discussions in Section 6.
TABLE 1: Material properties of a composite plate: mass density $(\rho)$, Young's modulus $(E)$, shear modulus $(G)$, Poisson coefficient $(v)$, and thickness $(t)$.

\begin{tabular}{lccccc}
\hline$\rho\left(\mathrm{kg} / \mathrm{m}^{3}\right)$ & $E_{x}(\mathrm{GPa})$ & $E_{y}(\mathrm{GPa})$ & $v$ & $G_{x y}(\mathrm{GPa})$ & $t(\mathrm{~mm})$ \\
\hline 1700 & 131 & 8.2 & 0.281 & 4.5 & 3 \\
\hline
\end{tabular}

\section{Delamination-Induced Standing Waves}

First, the existence of standing waves induced by an internal delamination in a composite structure is investigated through FE simulation. To simplify and clarify the problem, Lamb wave propagation along a composite plate is assumed in the simulation. Note that laser-generated ultrasonic waves propagating along a thin plate-like structure eventually make Lamb waves in the far-field from the excitation laser source [9]. A 2D composite plate model with an internal delamination is shown in Figure 1. The 2D plane strain model with fournode bilinear quadrilateral (CPS4R) elements is made by using ABAQUS/Standard 6.11 [10]. The 2D composite plate model has a dimension of $210 \times 3 \mathrm{~mm}^{2}$, and the dimension of an APC 850 type PZT [11] is $10 \times 0.508 \mathrm{~mm}^{2}$ as shown in Figure 1. Here, PZT is used for Lamb wave generation, and the corresponding responses are measured at all nodes across delamination in the time domain for visualizing the wave interactions with the delamination. The material properties of the composite plate are summarized in Table 1. Since 2D plane strain model is used in this simulation, four parameters are only considered due to the symmetricity.

To precisely investigate the Lamb wave interactions with the entrance and exit of delamination, the internal delamination is modeled under varying its width as shown in Figure 1. The width varying delamination is modeled using a doublenode [10], and the constraint conditions of the double-node between two delamination interfaces are defined as follows. For normal behavior, the delamination surfaces transmit contact stresses only when they are in contact, but no penetration is allowed at each constraint location. For tangential behavior, the relative sliding motion between the two surfaces is prevented as long as the corresponding normal contact constraints are active. The delamination widths are varied from 0 to $20 \mu \mathrm{m}$ as shown in Figure 1. The PZT attached on the top surface is used to generate Lamb waves by applying the input waveform of 7-cycle toneburst signals with a driving frequency of $100 \mathrm{kHz}$. To guarantee proper simulation results, the spatial and time resolution should be well designed. The mesh size of $0.5 \times 0.5 \mathrm{~mm}^{2}$ and the sampling rate of $20 \mathrm{MHz}$ are determined by the following spatial discretization rule [12]:

$$
\begin{aligned}
\max (\Delta x, \Delta y) & <\frac{\delta_{\min }}{10} \\
\Delta t & <\frac{0.7 \min (\Delta x, \Delta y)}{C_{L}},
\end{aligned}
$$

where $\Delta x, \Delta y$, and $\delta_{\min }$ represent $x$ and $y$ directional element dimensions and the shortest wavelength at a given frequency, respectively. $\Delta t$ denotes time interval. 


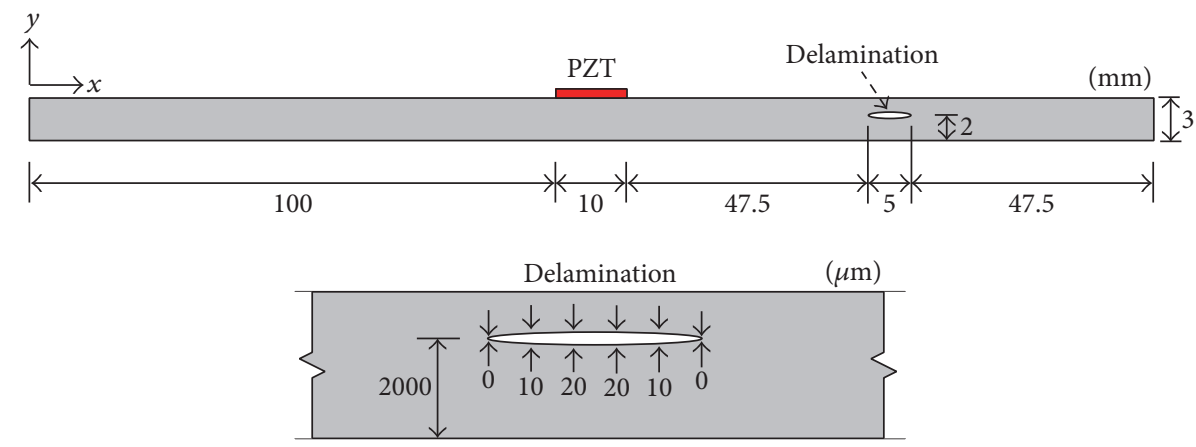

FIGURE 1: Description of 2D plane strain FE model.

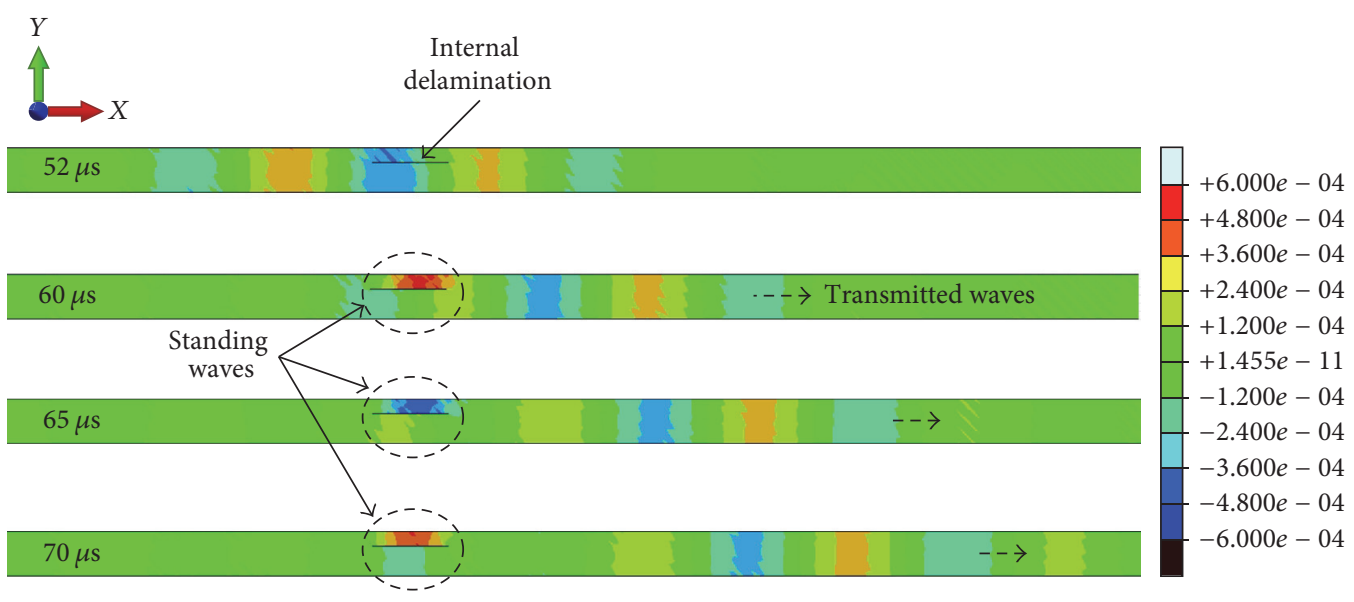

FiguRE 2: Representative out-of-plane velocities of Lamb wave propagation at $52 \mu \mathrm{s}, 60 \mu \mathrm{s}, 65 \mu \mathrm{s}$, and $70 \mu \mathrm{s}$.

The representative out-of-plane velocities of Lamb wave propagation at $52 \mu \mathrm{s}, 60 \mu \mathrm{s}, 65 \mu \mathrm{s}$, and $70 \mu \mathrm{s}$ are shown in Figure 2. Since the magnitude of fundamental antisymmetric $\left(A_{0}\right)$ modes is dominant rather than fundamental symmetric $\left(S_{0}\right)$ modes in this frequency range, the responses of Figure 2 mainly show $A_{0}$ Lamb wave modes. When $A_{0}$ modes propagating along the composite plate encounter the internal delamination, a portion of them is trapped within the delamination, and others are transmitted through the delamination. It is interesting to see here that the waves reflected from the delamination entrance are hardly observed. On the other hand, the delamination exits act as dominant reflectors once the waves go into the delamination boundary. Therefore, the trapped waves undergo multiple reflections from the delamination boundary, generating standing waves within the delamination. These standing waves can be effectively used as the strong evidence of the delamination existence.

\section{Zero-Lag Cross-Correlation (ZLCC) Imaging}

The ZLCC concept was firstly proposed by Zhu et al. for fast damage imaging based on the time reversal principle [13]. As the follow-up studies, the ZLCC concept has been applied to damage detection in composite [14] and metallic [15] structures. This section explains how ZLCC is calculated from the measured wavefield. In particular, the relationship between ZLCC and the standing waves caused by impactinduced delamination is thoroughly investigated. As the first step, total ultrasonic wavefield $\left(W_{T}\right)$ is measured in the timespace $(t-s)$ domain on the target area of interest. Here, $W_{T}$ typically includes wave propagation, wave interactions with delamination, and measurement noises. Once $W_{T}$ is obtained at all spatial points of interest, it can be decomposed into forward $\left(W_{F}\right)$ and backward $\left(W_{B}\right)$ propagating waves using the frequency-wavenumber $(f-k)$ domain analysis $[5,7,16$, 17]. $W_{T}$ in the $t$ - $s$ domain is transformed into the $f$ - $k$ domain using a 3D Fourier transform

$$
\begin{aligned}
& U_{T}\left(k_{x}, k_{y}, \omega\right) \\
& \quad=\iiint_{-\infty}^{\infty} W_{T}(x, y, t) e^{-i\left(k_{x} x+k_{y} y+\omega t\right)} d x d y d t,
\end{aligned}
$$

where $U_{T}$ is the ultrasonic wavefield in the $f-k$ domain. $k$ and $\omega$ are the wavenumber and angular frequency, respectively. $x$ and $y$ denote spatial coordinates.

Now, window functions, $\Phi_{F}$ and $\Phi_{B}$, are introduced so that forward $\left(U_{F}\right)$ and backward $\left(U_{B}\right)$ propagating waves are decomposed from $U_{T}$ in the $f-k$ domain

$$
U_{F(B)}\left(k_{x}, k_{y}, \omega\right)=U_{T}\left(k_{x}, k_{y}, \omega\right) \cdot \Phi_{F(B)} \quad \forall \omega,
$$


where

$$
\begin{gathered}
\Phi_{F}= \begin{cases}0 & k_{x}, k_{y} \leq 0 \\
1 & k_{x}, k_{y}>0\end{cases} \\
\Phi_{B}= \begin{cases}1 & k_{x}, k_{y}<0 \\
0 & k_{x}, k_{y} \geq 0 .\end{cases}
\end{gathered}
$$

Next, the resultant waves in the frequency-space $(f-s)$ domain are obtained using the following inverse 2D Fourier transform:

$$
\begin{aligned}
& V_{F(B)}(x, y, \omega) \\
& \quad=\frac{1}{2 \pi} \iint_{-\infty}^{\infty} U_{F(B)}\left(k_{x}, k_{y}, \omega\right) e^{i\left(k_{x} x+k_{y} y+\omega t\right)} d k_{x} d k_{y},
\end{aligned}
$$

where $V_{F}$ and $V_{B}$ are the forward and backward wavefields in the $f$-s domain.

Then, the ZLCC values at each spatial point are obtained using

$$
Z(x, y)=\int V_{F}(x, y, \omega) V_{B}^{*}(x, y, \omega) d \omega,
$$

where the superscript $*$ denotes the complex conjugate. Note that the ZLCC computation in the $f$-s domain is more effective than the $t$-s domain computation in terms of reducing the computational costs. The relationship between standing wave generation mechanism and the internal delamination is described in Section 2. Multiple reflections within the delamination boundary momentarily make standing waves, meaning that the two different directional waves have the same wavelength and frequency conditions inside the delamination boundary. Such conditions well satisfy the ZLCC condition which physically means that the similarity indicator of two waves is zero-delayd or in-phase [15]. Therefore, the ZLCC values will abruptly increase within the delamination boundary compared to the intact region.

Next, an additional denoising process is necessary to remove the undesired measurement noises which can cause false alarms. The essence of the denoising process is that a threshold value (TR) computed by extreme value statistics [18] is employed with respect to the values of ZLCC. The probability density function of the ZLCC values is estimated by fitting a Weibull distribution to the ZLCC populations, and TR corresponding to a one-sided $95 \%$ confidence interval is established. Finally, the values of ZLCC exceeding TR highlight impact-induced delamination without noise components. This denoising process physically means that delamination should be automatically visualized and emphasized in the final ZLCC image if the delamination is large enough to create standing waves beyond the measurement noise level.

\section{Experimental Description}

To experimentally validate the proposed technique for delamination visualization, a complete noncontact LUI system [7] is introduced first. Because the LUI system can generate

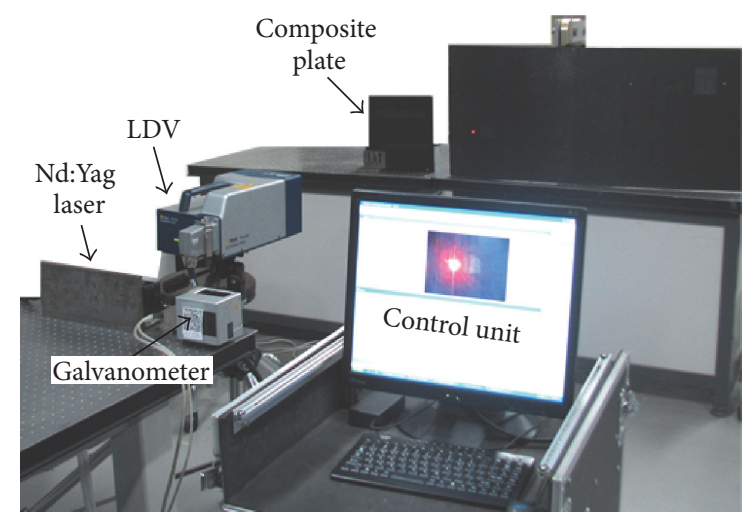

FIgURE 3: Experimental setup for delamination visualization in a composite plate.

and measure $W_{T}$ by scanning fully noncontact laser beams onto the target surface, no ultrasonic transducer installation is required.

Figure 3 shows the LUI system composed of a Q-switched Nd:Yag pulse laser for ultrasonic generation, LDV for ultrasonic measurement, a galvanometer for laser scanning, and a control unit. The overall working principle is as follows. First, virtual grid points on the target surface are created using a built-in digital camera, and the sequences of excitation and sensing scanning points are predetermined. Then, the controller in the control unit sends out a trigger signal to the $\mathrm{Nd}$ :Yag pulse laser to fire the excitation laser beam to the first prescribed excitation point through the galvanometer with a focal lens. The same trigger signal is simultaneously transmitted to LDV to activate data acquisition. Then, the response signal is measured at a specified measurement point, transmitted to, and stored in the control unit. Next, the control unit moves the excitation or sensing laser beam automatically to the next scanning point by sending control signals to the relevant galvanometer. By repeating the ultrasonic excitation and sensing over the prescribed grid points, $W_{T}$ can be constructed over the target surface.

The Q-switched Nd:Yag pulse laser employed in this system has $532 \mathrm{~nm}$ wavelength and $3.7 \mathrm{MW}$ peak power and generates a pulse input with $8 \mathrm{~ns}$ pulse duration at a repetition rate of $20 \mathrm{~Hz}$. The galvanometer has a maximum rotating speed of $5730^{\circ} / \mathrm{s}$, angular resolution of $6.6 \times 10^{-4^{\circ}}$, and an allowable scanning angle of $\pm 21.8^{\circ}$. The initial laser beam diameter emitted from the galvanometer is about $4 \mathrm{~mm}$. Because this beam size is relatively large to achieve high spatial resolution, the focal lens installed in front side of the galvanometer adjusts the beam size to $0.5 \mathrm{~mm}$ at the optical focal length of $2 \mathrm{~m}$. Ultrasonic waves are created through the thermal expansion of an infinitesimal area heated by the highpower laser. The power level, laser pulse duration, and laser beam size need to be carefully tailored because high-power density of the laser beam above a certain threshold will cause ablation phenomena [19].

For ultrasonic response measurement, a commercial scanning LDV (Polytec PSV-400-M4) with a built-in galvanometer and an autofocal lens is used in this system [20]. 

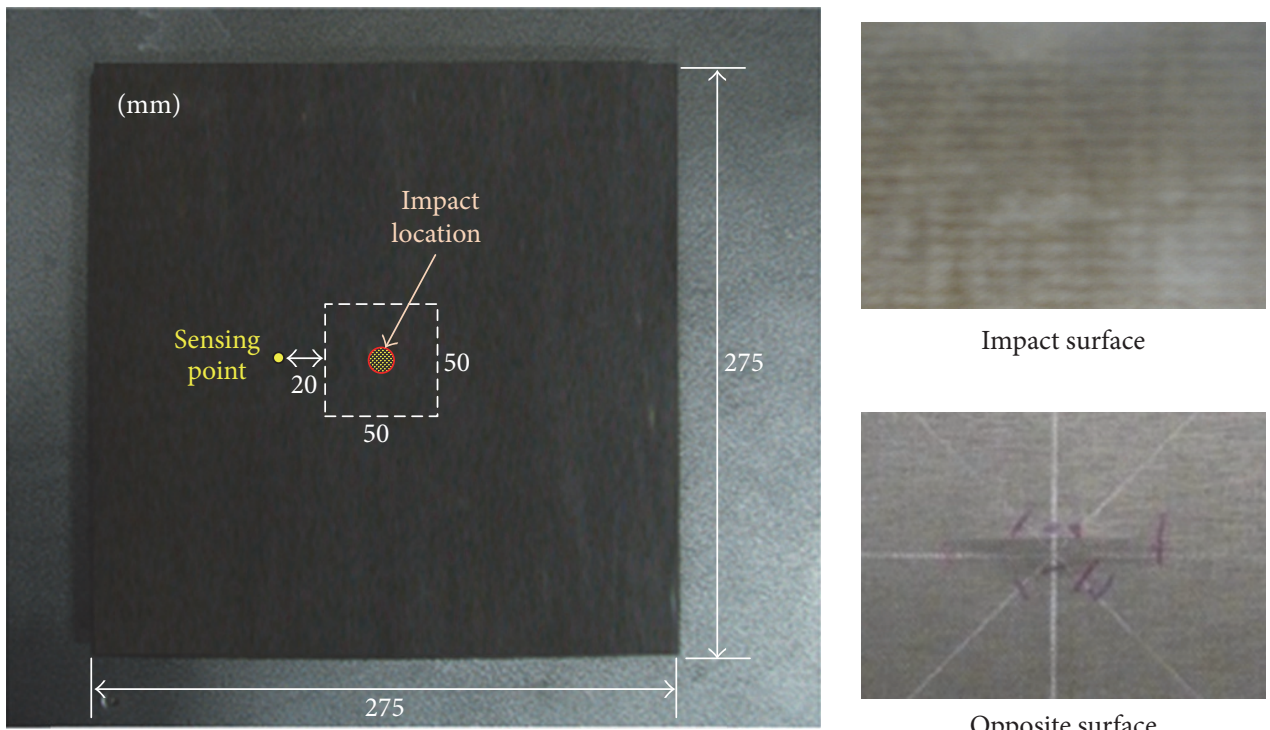

Opposite surface

FIGURE 4: Target composite plate with the impact-induced delamination and the laser scanning scheme.

The laser source used for LDV is the He-Ne laser with a wavelength of $633 \mathrm{~nm}$, and the optimal measurement distance is repeated itself at $99+204 n(\mathrm{~mm})$, where $n$ denotes an integer number. Then, the minimum focal length of the autofocal lens is $0.35 \mathrm{~mm}$, and the allowable scanning angle range and scanning speed are $\pm 20^{\circ}$ and $2000^{\circ} / \mathrm{s}$, respectively. This $1 \mathrm{D} \mathrm{LDV}$ measures the out-of-plane velocity in the range of $0.01 \mu \mathrm{m} / \mathrm{s}$ to $10 \mathrm{~m} / \mathrm{s}$ over a target surface based on the Doppler frequency-shift effect of light. Since the intensity of the signal laser beam reflected from the target surface highly depends on the surface condition, a special surface treatment is often necessary to improve the reflectivity of the returned laser beam.

The control unit consists of a personal computer (PC), controller, velocity decoder with the maximum velocity sensitivity of $1 \mathrm{~mm} / \mathrm{s} / \mathrm{V}$, and a 14-bit digitizer with a maximum sampling frequency of $350 \mathrm{kHz}$. The controller sends out trigger signals to launch the excitation laser beam and to simultaneously start the data collection. In addition, the controller generates control signals to aim the excitation and sensing laser beams at desired target positions. The velocity decoder records the out-of-plane velocity by computing the frequency shifts between the laser beam reflected from the target surface and the reference laser beam. Then, the measured signals are processed on PC.

Figure 4 shows the target composite plate with a dimension of $275 \times 275 \times 1.8 \mathrm{~mm}^{3}$. The composite plate is composed of IM7 graphite fibers with 977-3 resin material and consists of 12 plies with a layup of $[0 / \pm 45 / 0 / \pm 45] \mathrm{s}$. Then, the composite plate is subjected to impact, causing an internal delamination. The created delamination is invisible on the impact surface, but partially broken fibers can be observed on the opposite surface to the impact surface as shown in Figure 4. The laser scanning is performed on the impact surface, and an area of $50 \times 50 \mathrm{~mm}^{2}$ is scanned by the excitation laser beam with spatial resolution of $2 \mathrm{~mm}$. Then, the corresponding ultrasonic waves are measured by LDV at $20 \mathrm{~mm}$ apart from the left edge of the scanning area as shown in Figure 4. The distances from the Nd:Yag laser for scanning ultrasonic generation and LDV for ultrasonic measurement to the target specimen are $2 \mathrm{~m}$ and $1.6 \mathrm{~m}$, respectively. For the $\mathrm{Nd}$ :Yag laser, the repetition rate, peak power, and laser beam size are set to $20 \mathrm{~Hz}, 1 \mathrm{MW}$, and $4 \mathrm{~mm}$, respectively. Then, the sampling rate of $\mathrm{LDV}$ is $5.12 \mathrm{MHz}$, and the sensitivity of the velocity measurement is set to $10 \mathrm{~mm} / \mathrm{s} / \mathrm{V}$. A retroreflective tape is placed at the sensing point of the target specimen to improve reflectivity of the sensing laser beam. The response signals are measured 50 times for each excitation point, averaged in the time domain, and bandpass-filtered with $10 \mathrm{kHz}$ and $300 \mathrm{kHz}$ cutoff frequencies to improve signal-to-noise ratio.

\section{Experimental Results}

Once $W_{T}$ is collected from all scanning points of the composite plate using the LUI system, the $W_{T}$ image is obtained by assembling all $W_{T}$ data within the scanning area. Figure 5 shows the representative snapshots of $W_{T}$ obtained from the composite plate with delamination at $26.60 \mu \mathrm{s}, 34.80 \mu \mathrm{s}$, and $43.01 \mu \mathrm{s}$. Here, the amplitude of each snapshot is normalized with respect to its maximum value. It is clearly observed that incident waves propagating from left to right are trapped by interacting with the internal delamination, and others are mainly transmitted through the delamination rather than reflected from the delamination. The experimental observation is well matched with the numerical expectation described in Section 2.

Subsequently, $W_{T}$ is transformed to $U_{T}$ using the $3 \mathrm{D}$ Fourier transform in (2) and $U_{F}$ and $U_{B}$ are obtained using (3). The corresponding wavenumber plots are displayed in Figure 6. It can be easily observed that $U_{T}$ is successfully decomposed into $U_{F}$ and $U_{B}$. Note that the waves mainly 

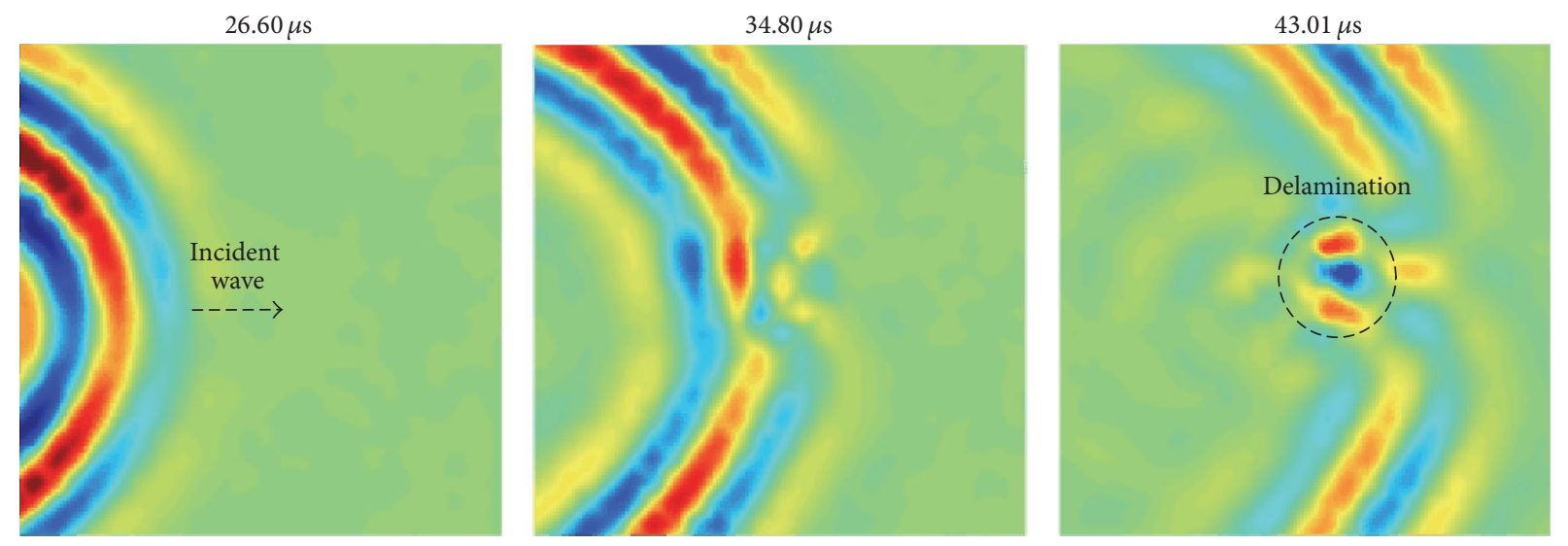

Figure 5: Representative $W_{T}$ images at $26.60 \mu \mathrm{s}, 34.80 \mu \mathrm{s}$, and $43.01 \mu \mathrm{s}$.
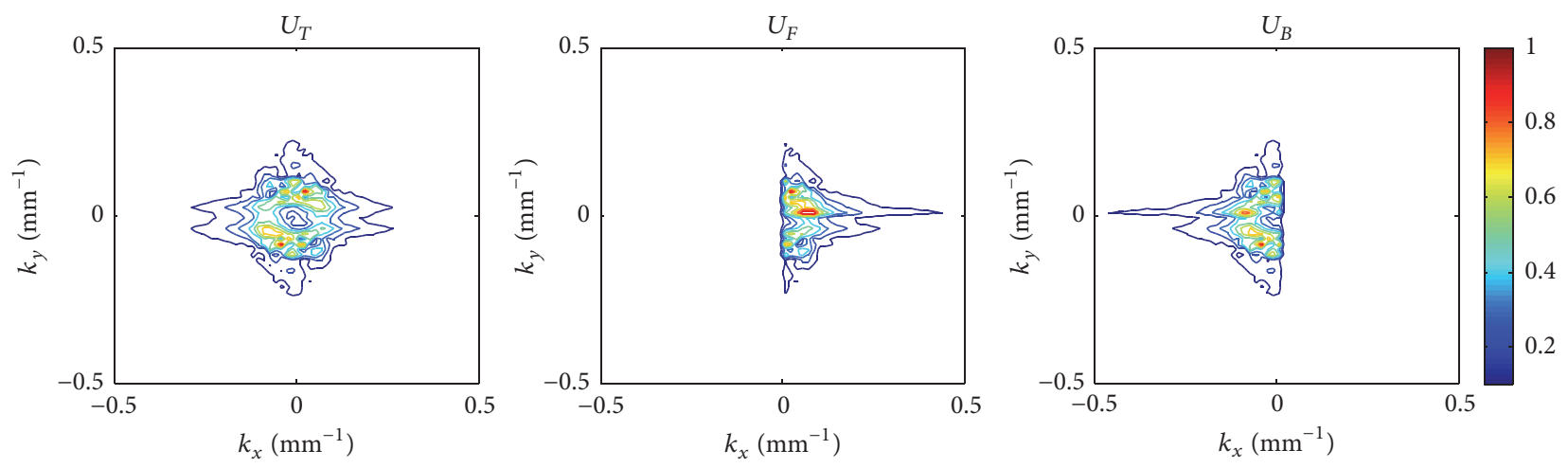

FIgURE 6: Wavenumber plots of $U_{T}, U_{F}$, and $U_{B}$.

propagate from left- to right-hand side in the inspection area of interest as observed in Figure 5.

In order to more precisely investigate the wave decomposition process, the decomposed $U_{F}$ and $U_{B}$ in the $f-k$ domain are transformed into $W_{F}$ and $W_{B}$ in the $t$-s domain, respectively, using the following inverse 3D Fourier transform:

$$
\begin{aligned}
& W_{F(B)}(x, y, t)=\frac{1}{2 \pi} \\
& \quad \cdot \iiint_{-\infty}^{\infty} U_{F(B)}\left(k_{x}, k_{y}, \omega\right) e^{i\left(k_{x} x+k_{y} y+\omega t\right)} d k_{x} d k_{y} d \omega .
\end{aligned}
$$

In (7), $W_{F}$ and $W_{B}$ contain only forward and backward propagating waves. The computed $W_{F}$ and $W_{B}$ images at $26.60 \mu \mathrm{s}, 34.80 \mu \mathrm{s}$, and $43.01 \mu \mathrm{s}$ are shown in Figures 7(a) and 7(b), respectively. Incident waves transmitted through the delamination are clearly observed in Figure 7(a) while only reflected waves from the delamination boundary are shown in Figure 7(b). The decomposed wavefields of Figure 7 reveal that the proposed wave decomposition process works well for $W_{T}$.

Based on the validation results, the ZLCC values are subsequently computed using (6). Then, the denoising process described in Section 3 is applied to all ZLCC values. Here, TR is computed as 0.578 . By assembling all ZLCC values exceeding TR at all spatial points of interest, the ZLCC image is obtained as shown in Figure 8(a). The dotted circle with around $10 \mathrm{~mm}$ diameter shows the actual delamination size estimated by the thermographic image shown in Figure 8(b). By comparing the same dotted circle in Figure 8(a) with 8(b), it is confirmed that the proposed ZLCC imaging technique has high accuracy for hidden delamination localization and size estimation. Again, the ZLCC image reveals that only delamination is identified, localized, and quantified without any comparison of baseline data previously obtained from the pristine condition of the composite plate, making it possible to minimize false alarms caused by operational and environmental variations by avoiding pattern comparisons with the ultrasonic wavefield images previously obtained from the pristine condition of a target structure [21].

\section{Conclusion}

This study proposes a complete noncontact laser ultrasonic scanned zero-lag cross-correlation (ZLCC) imaging technique for impact-induced delamination visualization. In particular, the wave interactions with invisible delamination are precisely analyzed by employing a finite element method, and the corresponding standing waves are visualized by ZLCC imaging. The experimental validation using a complete noncontact laser ultrasonic imaging system revealed that impact-induced delamination is successfully visualized 


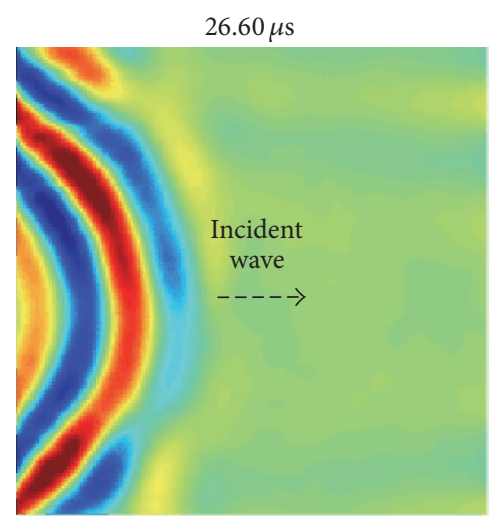

$26.60 \mu \mathrm{s}$
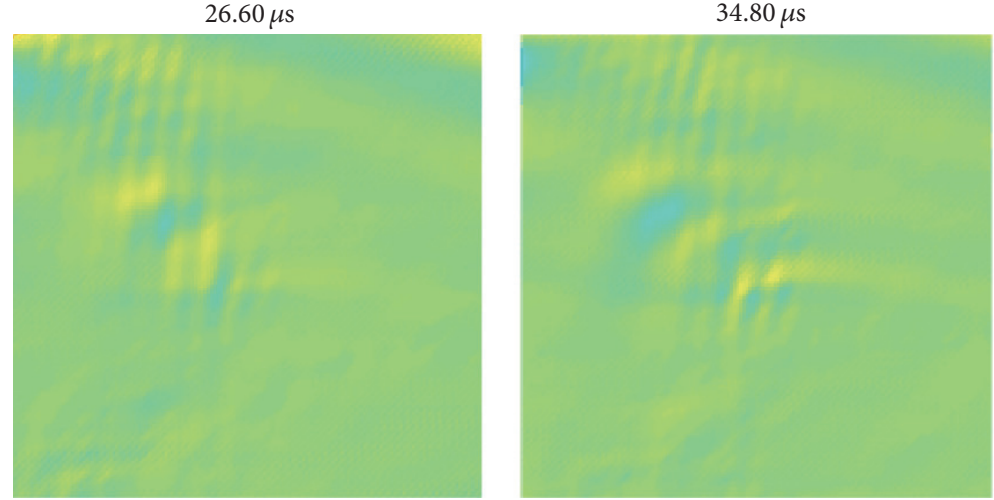

(b)

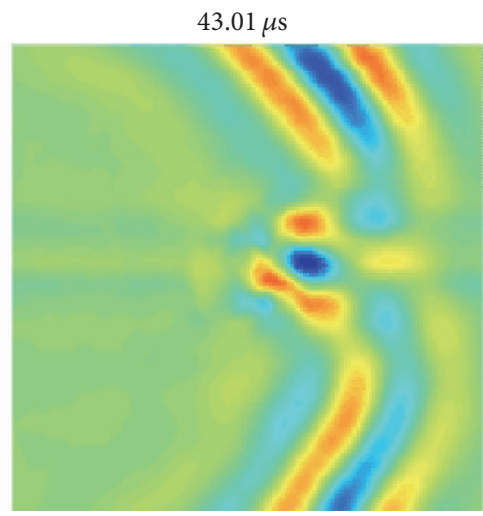

$43.01 \mu \mathrm{s}$

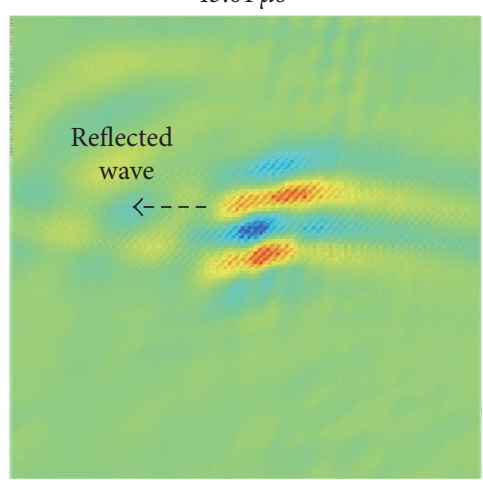

FIGURE 7: Decomposed wavefield images: (a) $W_{F}$ and (b) $W_{B}$ images.

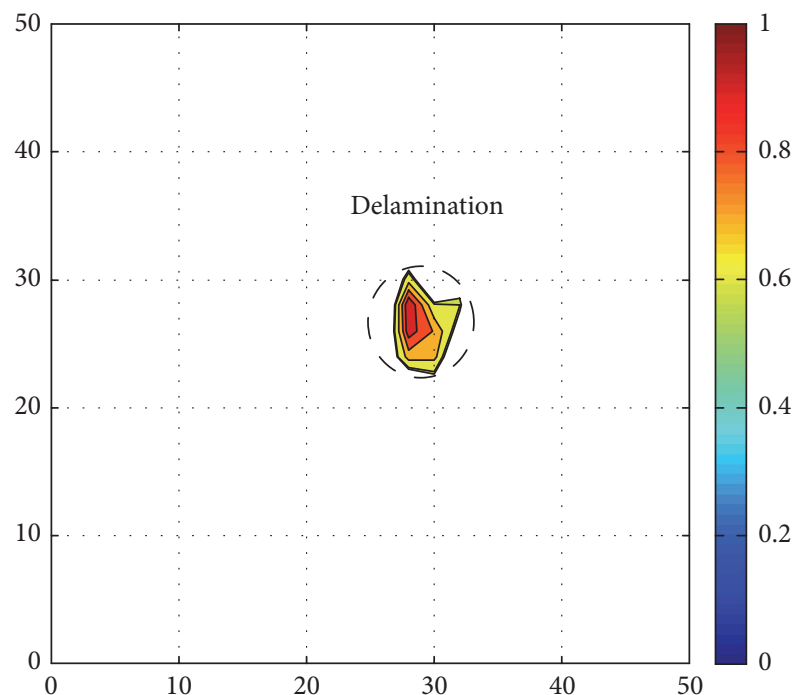

(a)

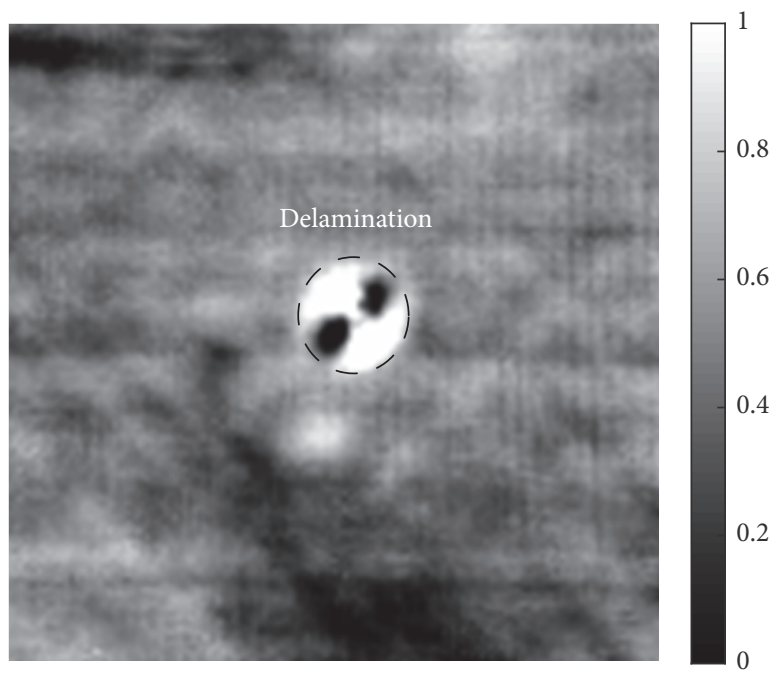

(b)

FIGURE 8: Automated delamination visualization results: (a) ZLCC image and (b) thermographic image.

without relying on baseline data, enabling minimization of false damage alarms due to changing operational and environmental conditions. Furthermore, fully automated damage diagnosis is accomplished without any users' intervention.
Although the ZLCC imaging technique is promising tool for hidden delamination identification, localization, and quantification, there are still some technical challenges associated with the sensing process for field applications: (1) because of 
the required high spatial resolution, the data collection may take a relatively long time; (2) a special treatment of a target surface is often necessary to enhance the reflectivity of the sensing laser beam; and (3) there can be the eye safety issue associated with the class 4 excitation laser although the class 2 sensing laser is known to be safe. Indeed, these technical hurdles are critical issues to be resolved before the proposed technique is applied to various real structures. The equipment improvement such as high spatial resolution ultrasonic camera or high performance multipoint sensing laser interferometry might be promising solution for the prescribed limitations. Further studies are warranted to address these issues.

\section{Disclosure}

Any opinion, finding, and conclusion or recommendation expressed in this material are those of the author and do not necessarily reflect the views of the funding agency.

\section{Competing Interests}

The author declares no competing interests.

\section{Acknowledgments}

This work is supported by Basic Science Research Program through the National Research Foundation of Korea (NRF) funded by the Ministry of Science, ICT and Future Planning (2015R1C1A1A01052625), and the experimental devices were supported by Professor Hoon Sohn at KAIST, Republic of Korea.

\section{References}

[1] X. Zhao, H. Gao, G. Zhang et al., "Active health monitoring of an aircraft wing with embedded piezoelectric sensor/actuator network: I. Defect detection, localization and growth monitoring," Smart Materials and Structures, vol. 16, no. 4, pp. 1208-1217, 2007.

[2] F. L. Di Scalea, H. Matt, I. Bartoli, S. Coccia, G. Park, and C. Farrar, "Health monitoring of UAV wing skin-to-spar joints using guided waves and macro fiber composite transducers," Journal of Intelligent Material Systems and Structures, vol. 18, no. 4, pp. 373-388, 2007.

[3] Y.-K. An, M. K. Kim, and H. Sohn, "Airplane hot spot monitoring using integrated impedance and guided wave measurements," Structural Control and Health Monitoring, vol. 19, no. 7, pp. 592-604, 2012.

[4] D. Dhital and J. R. Lee, "A fully non-contact ultrasonic propagation imaging system for closed surface crack evaluation," Experimental Mechanics, vol. 52, no. 8, pp. 1111-1122, 2012.

[5] Y.-K. An, B. Park, and H. Sohn, "Complete noncontact laser ultrasonic imaging for automated crack visualization in a plate," Smart Materials and Structures, vol. 22, no. 2, Article ID 025022, 2013.

[6] C. C. Chia, J.-R. Lee, C.-Y. Park, and H.-M. Jeong, "Laser ultrasonic anomalous wave propagation imaging method with adjacent wave subtraction: application to actual damages in composite wing," Optics \& Laser Technology, vol. 44, no. 2, pp. 428-440, 2012.

[7] B. Park, Y.-K. An, and H. Sohn, "Visualization of hidden delamination and debonding in composites through noncontact laser ultrasonic scanning," Composites Science and Technology, vol. 100, pp. 10-18, 2014.

[8] M. S. Harb and F. G. Yuan, "Non-contact ultrasonic technique for Lamb wave characterization in composite plates," Ultrasonics, vol. 64, pp. 162-169, 2016.

[9] Y.-K. An, Y. Kwon, and H. Sohn, "Noncontact laser ultrasonic crack detection for plates with additional structural complexities," Structural Health Monitoring, vol. 12, no. 5-6, pp. 522-538, 2013.

[10] ABAQUS/Standard 6.11, User's Manual, Dessault Systems, Vélizy-Villacoublay, France, 2011.

[11] http://www.americanpiezo.com/.

[12] O. Diligent, Interaction between fundamental Lamb modes and defects in plates [Ph.D. thesis], Imperial College of Science, London, UK, 2003.

[13] R. Zhu, G. L. Huang, and F. G. Yuan, "Fast damage imaging using the time-reversal technique in the frequency-wavenumber domain," Smart Materials and Structures, vol. 22, no. 7, Article ID 075028, 2013.

[14] J. He and F.-G. Yuan, "Damage identification for composite structures using a cross-correlation reverse-time migration technique," Structural Health Monitoring, vol. 14, no. 6, pp. 558570, 2015.

[15] M. S. Harb and F. Yuan, "Damage imaging using non-contact air-coupled transducer/laser Doppler vibrometer system," Structural Health Monitoring, vol. 15, no. 2, pp. 193-203, 2016.

[16] M. Ruzzene, "Frequency-wavenumber domain filtering for improved damage visualization," Smart Materials and Structures, vol. 16, no. 6, pp. 2116-2129, 2007.

[17] T. E. Michaels, J. E. Michaels, and M. Ruzzene, "Frequencywavenumber domain analysis of guided wavefields," Ultrasonics, vol. 51, no. 4, pp. 452-466, 2011.

[18] H. W. Park and H. Sohn, "Parameter estimation of the generalized extreme value distribution for structural health monitoring," Probabilistic Engineering Mechanics, vol. 21, no. 4, pp. 366-376, 2006.

[19] C. B. Scruby and L. E. Drain, Laser Ultrasonics: Techniques and Applications, Taylor \& Francis, London, UK, 1990.

[20] http://www.polytec.com.

[21] Y.-K. An and H. Sohn, "Instantaneous crack detection under varying temperature and static loading conditions," Structural Control \& Health Monitoring, vol. 17, no. 7, pp. 730-741, 2010. 

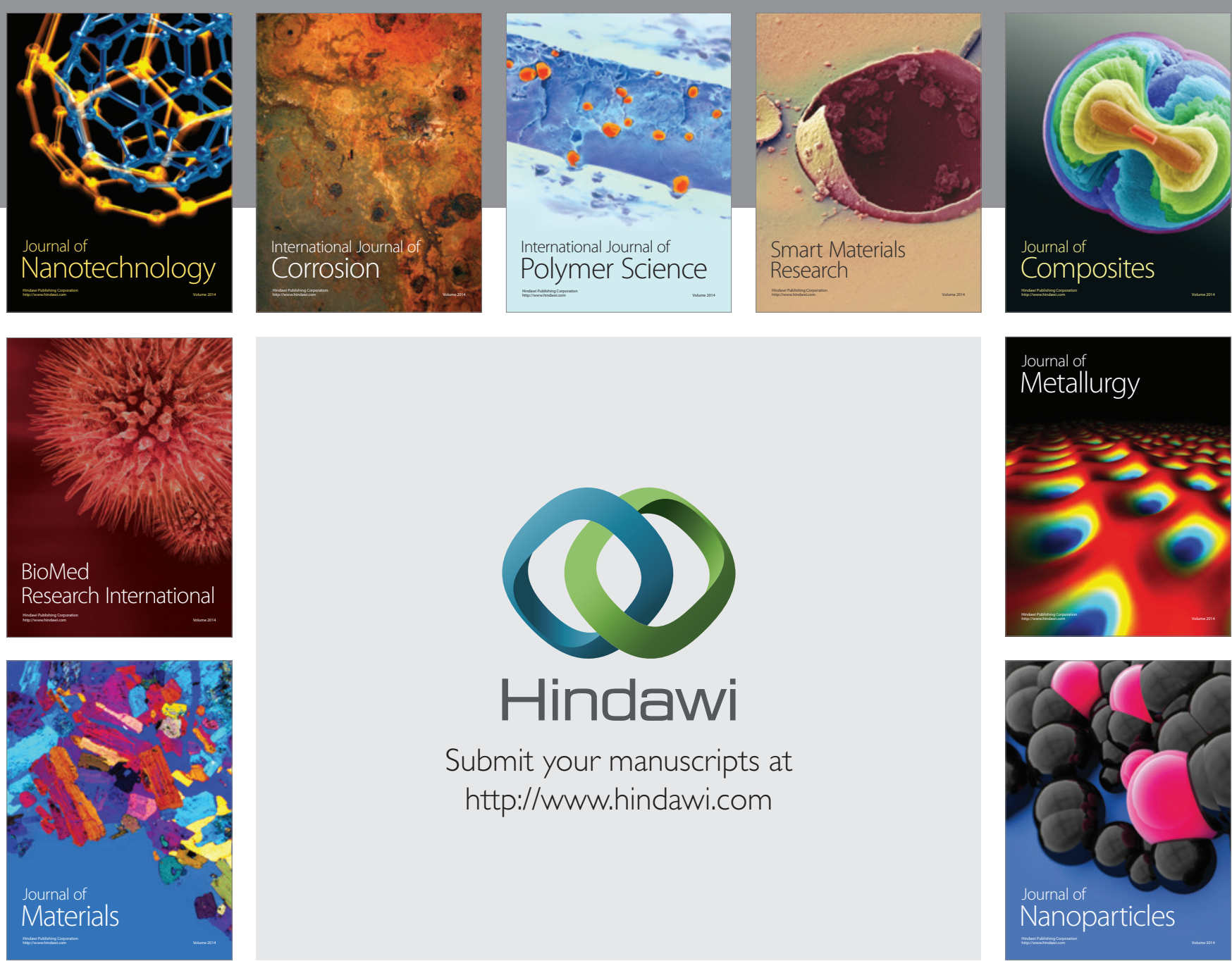

\section{Hindawi}

Submit your manuscripts at

http://www.hindawi.com

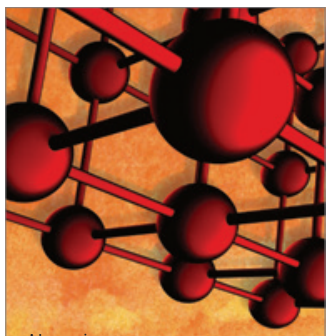

Materials Science and Engineering
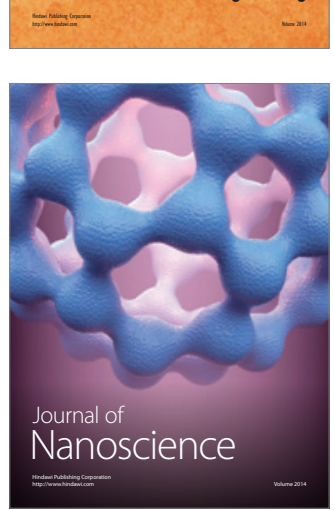
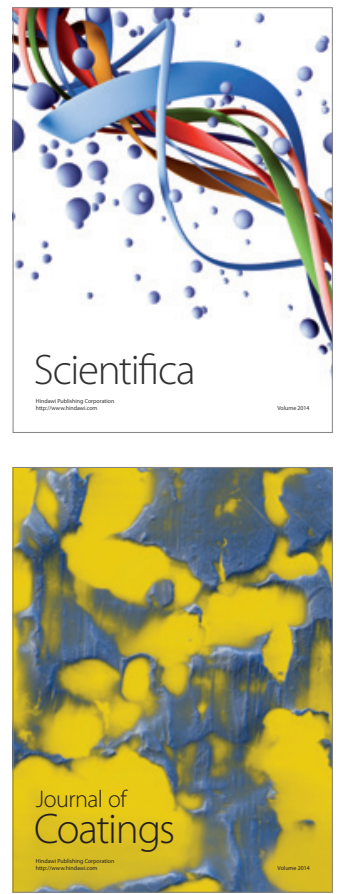
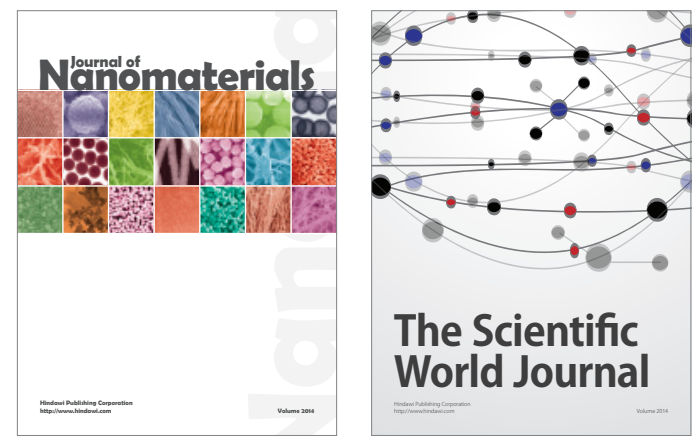

The Scientific World Journal
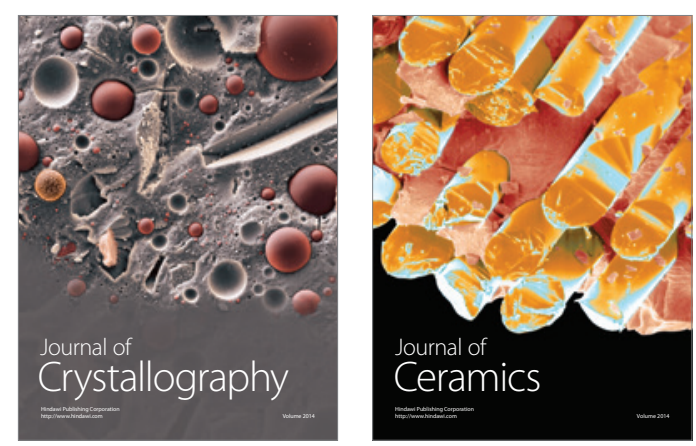
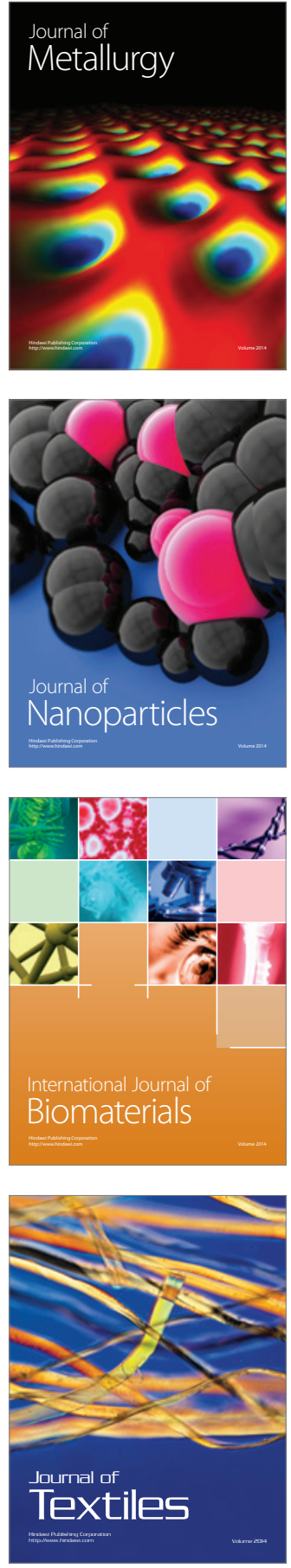